\title{
RADIOATTIVITÃ DI UNA LAVA ETNEA STUDIO QUANTITATIVO
}

\author{
L. Barbera, M. Curatolo *, M. M. Indovina Addario, \\ D. Palumbo, M. Santangelo
}

1. Introduzione. - Iu un lavoro recentemente publblicato $\left(^{1}\right)$ ablbiamo riportato i risultati di alcune misure di altività u eseguite su lave dell'Etna appartenenti ad eruzioni arvenute in epoche diverse. La tecnica impiegata $\grave{e}$ stata quella delle sezioni levigate sorrapposte ad emulsioni nucleari, da molti autori già descritta.

Proseguendo in questo campo di ricerche abljiamo tentato di mettere a punto un metodo quantitativo per la determinazione del rapporto delle concentrazioni di Uranio e 'Torio, $C_{\mathrm{V}} / C_{\mathrm{Th}}$, nei materiali a basso contenuto radioatlivo.

Abliamo pure cercato di studiare il problema della determinazione dell'equilibrio per la famiglia dell'Uranio, in vista dell'interesse che questo problema presenta in relazione alle questioni riguardanti i processi di formazione e di differenziazione della crosta terrestre.

Di recente $(-)$ sono stati sviluppati alcuni calcoli che perrengono a semplici formule di facile applicazione, atti alla determinazione, disponendo di una statistica sufficientemente ricca dei valori dei percorsi delle particelle s, della concentrazione nel minerale di ciascuna specie di nuclide emittente particelle $u$. E possibile inoltre, partendo dai suddetti calcoli, rilevare l'esistenza o meno dell'equilibrio radioattivo delle faniglie presenti nel materiale.

In questo primo lavoro quantitativo, disponendo di una statistica su circa 2000 tracce, noi useremo per la determinazione di $C_{V} / C_{T h}$ un metodo grlobale, hasalo sui calcoli sopra accennati, che pensiamo possa adattarsi meglio al caso da noi studiato.

2. Metodi di misura. - La tecnica sperimentale ormai classica consiste nella esposizione di lastre per fisica nucleare al materiale emittente particelle $u$, che nel nostro caso consisteva in un frammento di

$\left(*^{*}\right)$ Istituto di Mineralogia, Universita, Palermo. 
lava dell'Etna, appartenente ad una colata della eruzione dell'anno 1928. Dello stesso campione era stata precedentemente determinata l'attività specifica globale $\left({ }^{1}\right)$, a differenza però di quanto era stato fatto allora, abbiamo preferito sovrapporre alla lastra anziché una sezione levigata della lava un agglomerato di polvere della medesima. È questo un metodo già impiegato da diversi autori $\left({ }^{3}\right),\left({ }^{4}\right),\left({ }^{5}\right)$ per ottenere uno strato sufficientemente omogeneo sia in superficie che in profondità. (Un indizio del conseguimento di tale omogeneità può essere dedotto dal fatto che sulla lastra esposta abbiamo rilevato una distribuzione sensibilmente normale delle tracce $\alpha$ ).

Lo spessore dello strato di polvere che copriva l'emulsione avera valore maggiore del range massimo delle $"$; si può quindi nei nostri calcoli considerare tale spessore come infinito rispetto al percorso delle $\alpha$.

Le lastre usate, Ilford $C_{2}$ spessore $50 \mu$, sono state esposte per 51 giorni.

Le modalità di conservazione delle lastre e le caratteristiche dei bagni di sviluppo, fissaggio, ecc. sono quelle descritte nel lavoro citato $\left({ }^{1}\right)$.

La superficie esposta è stata divisa in 2 parti che sono state esplorate indipendentemente con due miscroscopi diversi: sono state misurate le proiezioni orizzontali e quelle verticali delle tracce, l'approssimazione era di $0,4 u$ per le prime, di 1 u per le seconde: e poiché abbiamo constatato una buona coerenza tra i 2 gruppi di misure, abbiamo in ultimo mescolato tutti i dati ed eseguito i calcoli sul totale delle tracce.

Per trasformare le lunghezze delle tracce nell'emulsione, misurate al microscopio, in $\mathrm{cm}$ di aria eq. si è tenuto conto del fattore di contrazione della emulsione, che nel nostro caso è risultato da una misura diretta 2,4 , e della variazione del coefficiente di riduzione aria-gelatina in funzione della energia residua delle particelle. Quest'ultima correzione è stata calcolata in base ai dati sperimentali riportati da M. Bogaardt e B. Koudijs $\left({ }^{\circ}\right.$ \%

A questo punto non ci sembra superfluo rilevare che solitamente viene consigliato $(*),(T)$ di prendere un coefficiente di riduzione ariagelatina costante e precisamente per le $\mathrm{C}_{2}$ Ilford il valore 1750 , invece come si rileva dal confronto tra le curve sperimentali percorso in gelatina-energia e quelle percorso in aria-gelatina $\left({ }^{8}\right),\left({ }^{8}\right)$, il coefficiente di riduzione $\grave{e} u n a$ funzione crescente della energia residua. 
Assumendo per tale coefficiente il valore costante di 1750 si introdurrebbero errori per eccesso dell'ordine del $10 \%$ per le tracce corte ed errori per difetto dell'ordine del $3 \%$ per le più lunghe.

E probabile che correzioni dello stesso genere dovrebbero essere apportate nella valutazione del percorso delle particelle nel campione; questo introdurrebbe correzioni nelle formule applicate: di queste però non abbiamo potuto tenere conto per la mancanza di dati sperimentali. Si può comunque prevedere che tali correzioni sarehbero sensibili solo sulle tracce più corte; è per questa ragione, e per altri motivi di cui verrà detto in seguito, che $i$ calcoli sono stati eseguiti evitando di utilizzare le tracce corte.

3. Risultati. - Per poter applicare le formule [vedi bibl. (2)] è necessario determinare, nell'ipotesi abbastanza fondata che gli elementi altivi presenti siano essenzialmente quelli delle famiglie dell'Úranio e del Torio, il numero di tracce presenti nella emulsione aventi percorso o proiezione orizzontale (in $\mathrm{cm}$ d'a. eq.) maggiore di $R_{\mathrm{i}}$, essendo $R_{1}, \ldots R_{i} \ldots \ldots R_{p}$ i percorsi dei diversi gruppi di a delle due famiglie disposti in ordine crescente.

Riportiamo nella tabella $I$ i valori di $R_{i}$ corrispondenti agli elementi attivi sottosegnati anche perché questi dati, che riteniamo i più atlendibili ( $s$, differiscono un po da quelli riportati da altri autori in lavori di questo genere.

TABELIA I

\begin{tabular}{|c|c|c|c|c|c|c|c|c|c|c|c|c|c|c|c|}
\hline R in em aria & 2,65 & 2.70 & 3.18 & 3,21 & 3,30 & 3,81 & 3,98 & 1,05 & 1,28 & 1,68 & $1,73$. & 5.0 & 3,64 & 6,91 & 8,57 \\
\hline $\begin{array}{l}\text { Elementi della } \\
\text { famiglia dell-U }\end{array}$ & $\mathrm{U}_{1}$ & & Io & $U_{\mathrm{u}}$ & $\mathrm{Ra}$ & Po & & $R n$ & & $\operatorname{la} \mathbf{A}$ & & & & $\mathrm{RaC}$ & \\
\hline $\begin{array}{l}\text { Elementi delln } \\
\text { famiglia del Th }\end{array}$ & & Th & & & & & Ra 'Th & & 'Th $\mathrm{X}$ & & 'Th C & $T_{n}$ & Th A & & Th C \\
\hline
\end{tabular}

Nella tabella $I I$ sono riportati i numeri $N$ di tracce aventi proiezioni orizzontali comprese nell'intervallo corrispondente sopra segnato.

Per ridure al minimo gli errori di conteggio si sono prese in considerazione solo le tracce aventı proiezione orizzontale maggiore di 
$0,8 \mathrm{~cm}$. d'a., non è però escluso che qualcuna delle tracce avente proiezione di questo ordine sia andata perduta.

\section{TiBrLla II}

\begin{tabular}{|c|c|c|c|c|c|c|c|c|}
\hline r & $0,81-2,50$ & $2,21-2,51$ & $2,66-3,18$ & $3,19-3,21$ & $3,22-3,30$ & $3,1-3,84$ & $3,85-3,98$ & $3,99-1,95$ \\
\hline$\pi$ & 1209 & i9 & 269 & 10 & 35 & 146 & 32 & 11 \\
\hline r & $4,06-4,28$ & $4,29-4,66$ & $4,67-4,73$ & $4,74-5,00$ & $5,01-5,64$ & $5,65-6,91$ & $6,92-18,57$ & \\
\hline$N$ & 45 & 40 & 17 & 23 & 56 & 38 & 21 & \\
\hline
\end{tabular}

In figura 1 (tratto continuo, scala semilogarituica) è riportato in ordinate il numero di tracce aventi proiezione orizzontale maggiore della corrispondente ascissa, numero ricavato dai dati della tabella II. In detta figura sono esatti i valori delle ordinate relative

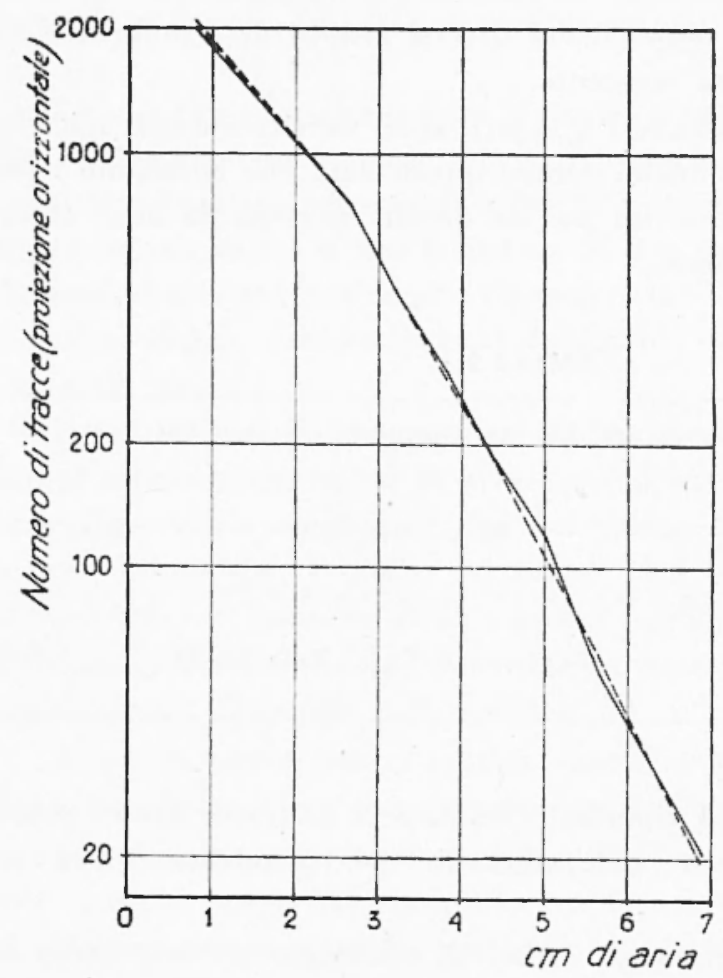

Fig. 1 ad ascisse unuali ai ranges dei vari gruppi di $u$, gli altri punti sono interpolati grafcamente.

ll caso della distribuzione delle lunghezze effeltive presenta maggiori difficoltà. Infatti scartando le tracce aventi proiezione orizzontale minore di $0,8 \mathrm{~cm}$ non si opera sulle lunghezze un vero taglio perché evidentemente si tralasciano, cosi facendo, quelle tracce che pur avendo proiezione 0 rizzontale molto piccola, il che rende difficile la loro identifi- 
cazione, possono avere lunghezza effettiva rilevante a causa di un alto valore della proiezione verticale. Ne viene che il numero di tracce aventi lunghezza effettiva magrgiore di $0,8 \mathrm{~cm}$ d'a. eq. sarà molto superiore a quello delle tracce aventi proiezione orizzontale maggiore di detlo valore. Si può però prevedere che le tracce aventi lunghezza maggiore di $2,65 \mathrm{~cm}$ d'a. eq. siano prevalentemente da ricercare tra quelle aventi proiezione orizzontale maggiore di $0,8 \mathrm{~cm}$ essendo relativamente rare le particelle 4 emesse quasi verticalmente.

In fig. 2 sono riporlati i risultati delle misure delle lunghezze tolali; per quanto detto sopra il grafico è attendibile solo a partire da valori delle ascisse non troppo piccole, mentre l'ordinata corrispondente a 0,8 $\mathrm{cm}$ dell'ascissa ha valore semplicemente indicalivo, ma notevolmente inferiore al vero.

Noliamo incidentalmente a questo proposito che la difficolta di scelta del valor minimo per le proiezioni orizzontali riduce noterolmente l'utilitia dello spettro delle lun.

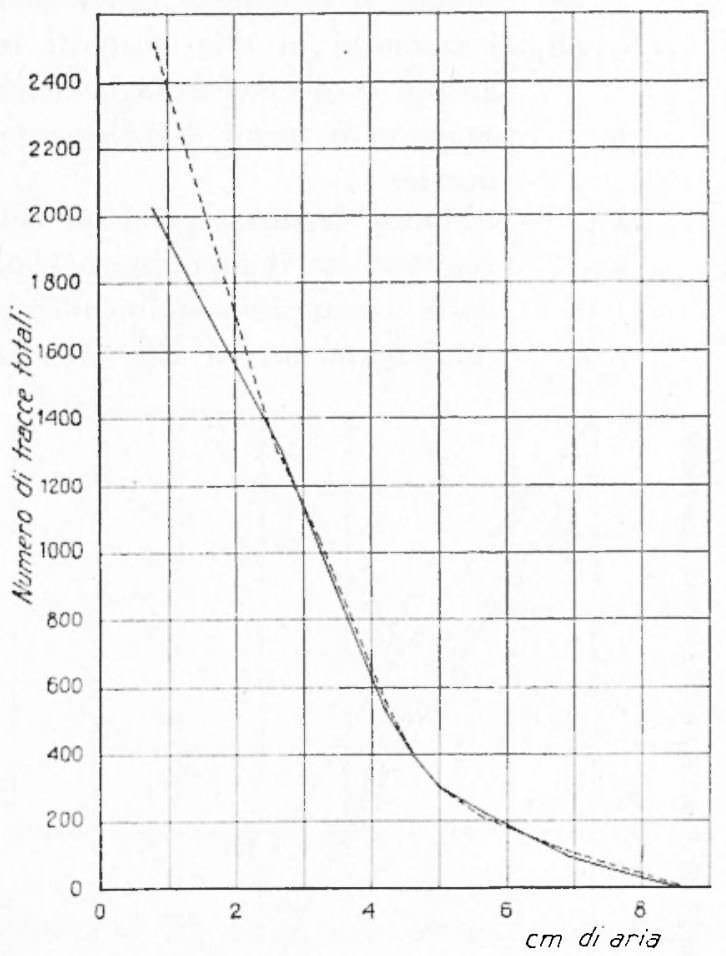

Fig. 2 ghezze effellive, selbene i calcoli su tale spettro siano molto più agevoli che su quello delle proiezioni orizzontali. Si arrebbero infatti inevitabilmente delle noteroli incertezze sul numero di tracce corte che sono quelle che più pesano per potere arrivare a qualche conclusione in merito all'equilibrio degli elementi componenti la famiglia dell'Uranio.

4. Interpreazione dei risultati. - Nei citali lavori $\left({ }^{2}\right)$ per il caso particolare di una famiglia in equilibrio, si perviene alle formule: 


$$
\begin{aligned}
& h(r)=\frac{\lambda n !}{4} \sum_{\mathrm{i}+1}^{\mathrm{p}}\left(R_{\mathrm{k}}-r\right) \varrho_{\mathrm{k}} \\
& g(\boldsymbol{r})=\frac{\lambda n \mu}{4} \sum_{\mathrm{i}+1}^{\mathrm{p}} \frac{\left(R_{\mathrm{k}}-r\right)^{\mathrm{e}}}{R_{\mathrm{k}}} \varrho_{\mathrm{k}}
\end{aligned}
$$

col seguente significato dei simboli:

$h(r), g(r)$ numero di tracce per $\mathrm{cn}^{-}$di emulsione e per secondo di esposizione aventi rispettivamente proiezione orizzontale o lunghezza totale nella gelatina $>r$;

$R_{1} \ldots R_{\mathrm{i}} \ldots R_{1}$; i cammini in aria, disposti in ordine crescente, dei gruppi di $\alpha$ emesse dagli elementi della famiglia;

$n \quad$ numero di atomi dell'elemento padre per $\mathrm{cm}^{3}$ di minerale;

$\lambda$ costante di disintegrazione dell'elemento padre;

$\mu \quad$ rapporto tra il cammino nel materiale e quello in aria delle « (supposto indipendente dall'energia); coefficiente dovuto alle eventuali biforcazioni.

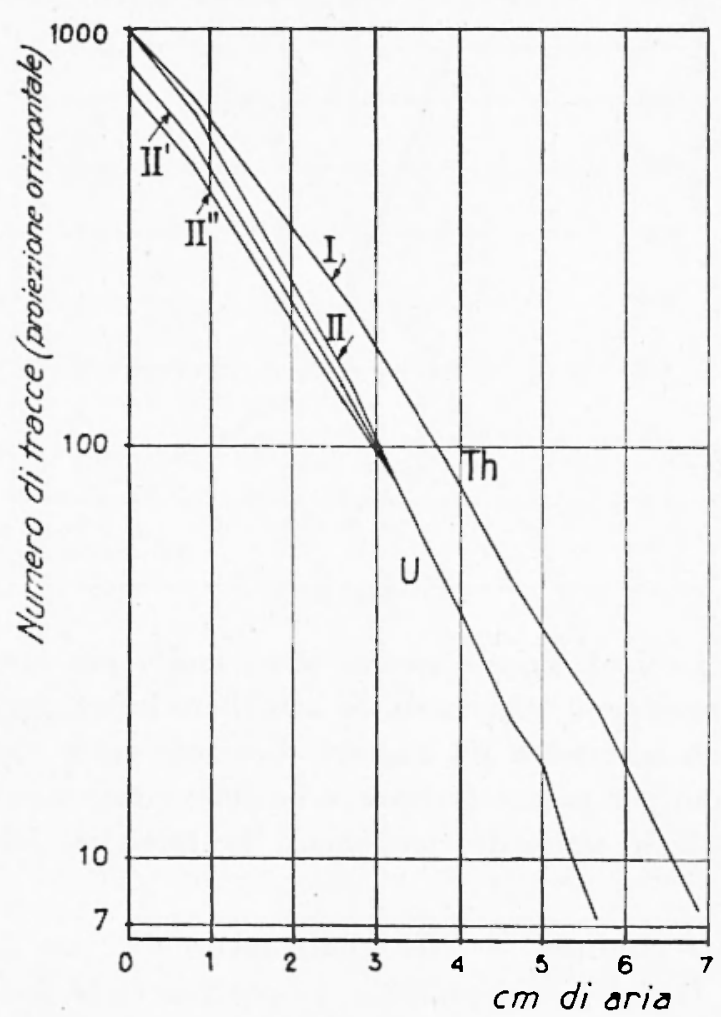

Fig. 3

Con ottima approssimazione $e=1$ per tutti gli elementi della famiglia dell'Uranio 238, per quella del Torio $\varrho \simeq 1 / 3$ per il ThC, e $2 / 3$ per il ThC', 1 per tutti gli altri elementi.

Partendo da queste formule sono stati costruiti i grafici delle figg. 3 e 4 nei quali sono riportati gli spettri integrali teorici delle proiezioni orizzontali, fig. 3 (scala semi logaritmica) e quelli delle lunghezze totali (fig. 4). In entrambe le figure la linea I si riferisce al caso di un materiale contenente solo Th in 
equilibrio con i suoi discendenti, la linea II al caso del solo $U^{238}$ anche esso in equilibrio, la $\mathrm{II}^{\prime}$ si riferisce alla presenza del solo Io in equilibrio ed infine la $\mathrm{II}^{\prime \prime}$ è nell'ipotesi che sia presente nel materiale il solo $\mathrm{Ra}$ in equilibrio con $\mathrm{i}$ propri discendenti. I dati sono stati calcolati per un totale di 1000 tracce per secondo e per $\mathrm{cm}^{2}$ e solo per i punti di ascisse uguali ai ranges dei vari gruppi di $\iota$ delle due famiglie, gli altri punti sono stati interpolati graficamente.

Nei calcoli che seguono sono stati utilizzati naturalmente solo $\mathrm{i}$ valori calcolati in base a [1] e [2] e non a quelli interpolati.

Nella lava in esame il Th sarà quasi certamente in equilibrio con i suoi discendenti poiché le vite medie di quest'ultimi sono di molto inferiori a quella del Th. Per quanto riguarda la famiglia dell'U si potrebbero avere invece difetti di equilibrio in corrispondenza dello Io o del Ra per ragio-

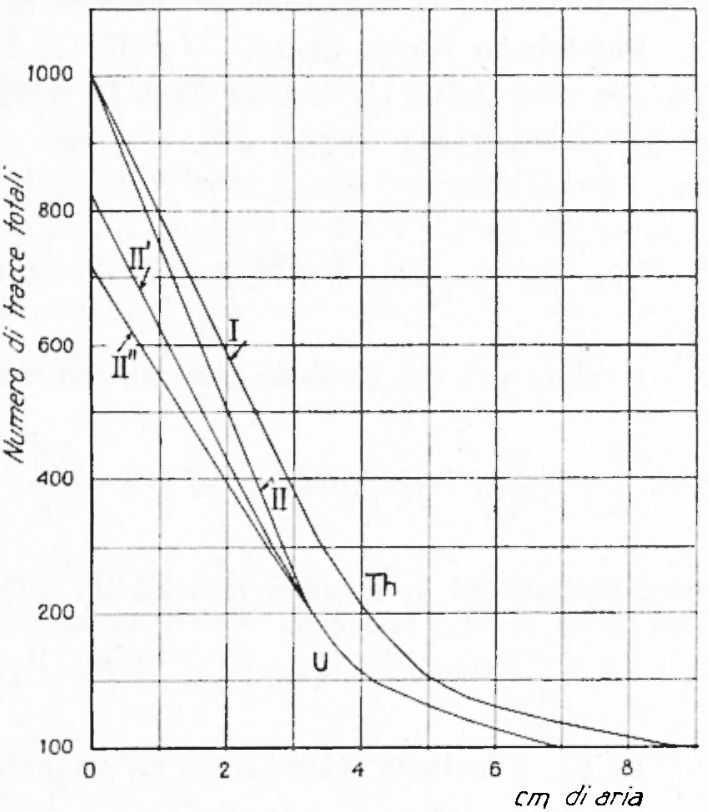

Fig. 1 ni chimiche o fisiche; il Ra però può analogamente al Th esser considerato in equilibrio secolare con gli elementi che lo seguono nella famiglia.

Abbiamo effettuato il confronto degli spettri sperimentali (figg. 1, 2) con quelli teorici (fig. 3,4) limitalamente a quelle parti delle due famiglie che possono supporsi in equilibrio, ossia, il che è equivalente, per valori delle ascisse maggiori di $3,21 \mathrm{~cm}$.

Indicando con $\varphi(r)$ le ordinate della linea $\mathrm{I}$, con $\%(r), \chi^{\prime}(r)$, $\chi^{\prime \prime}(r)$ quelle delle linee $\mathrm{II}, \mathrm{II}^{\prime}$ e $\mathrm{II}^{\prime \prime}$, con $f(\boldsymbol{r})$ le ordinate della curva sperimentale, con $a$ e $b$ le concentrazioni in unità convenzionali di Th e di Ra presenti nella lava, dovrà essere:

$$
f(\boldsymbol{r})=a \varphi(\boldsymbol{r})+b \%(r) \quad(3,21<\mathbf{r}<8,57)
$$


la [3] deve essere valida sia per gli spettri integrali delle lunghezze effettive (figg. 2,4 ) che per quelle delle proiezioni (figg. 1,3 ) ("i.

La determinazione di $a, b$ i stala eseguita tenendo conto del solo spettro delle proiezioni orizzontali che, come è stato notato sopra, è da ritenere più accuralo, e poiché a causa di inevitabili errori di osservazione non è possibile trovare 2 valori per $a$ e $b$ tali che la [3] venga verificata per tutti i valori $r$, si è preferito usare per la determinazione di $a$ e $b$ il metodo dei minimi quadrati.

Indichiamo con $r_{\mathrm{i}}, x_{\mathrm{i}}, f_{\mathrm{i}},(i=1, \ldots 11)$ i valori di $p(r), \chi(r)$, $f(r)$ per $r=3,21 ; \ldots .6,91 ; 8,57$, lo scarto quadratico $S(a, b)$ tra tra la distribuzione teorica [3] e quella sperimentale, è dato da:

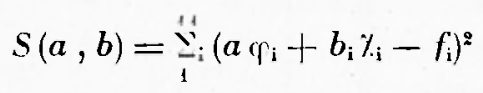

I valori $\bar{a}, \bar{b}$ che rendono minimo $S$ ottenuti risolvendo il sistema :

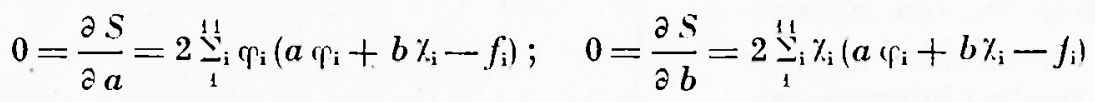

sono, usando per $f_{1}$ i ralori ricavati da Tal. II:

$$
\bar{a}=2,8 \quad \bar{b}=0,4
$$

In fig. 1 la linea tratteggiata rappresenta la distribuzione teorica partendo dai suddetti valori di $\vec{a}$ e $\bar{b}$. Come si vede l'accordo tra la distribuzione sperimentale e quella teorica è molto soddisfacente; per quanto riguarda lo spettro delle lunghezze totali lo speltro teorico (linea tratteggiala) si accorda pure abhastanza bene con quello sperimentale. In verità per quest'ultimo caso si avreble un accordo migliore prendendo per $\bar{a}$ un valore leggermente inferiore (circa 2,6); comunque questa piccola differenza carle entro gli errori sperimentali.

Le concentrazioni di Torio ed Uranio, $\mathrm{C}_{\mathrm{Th}}^{\circ}, \mathrm{C}_{\mathrm{U}}^{\circ}$, che darebbero luogo alle distribuzioni teoriche delle tracce come in figg. 3,4 (linee

${ }^{\left({ }^{3}\right)}$ A titolo di verifica della attendibilita dei risultati sperimentali è stato fatto un confronto tra lo spettro delle proiezioni orizzontali e quello delle proiezioni verticali: come era stato previsto $(-2)$ i due spettri si accordano in maniera soddisfacente. 
I e II) possono essere calcolate in $\mathrm{gr} / \mathrm{cm}^{3}$ di sostanza, mediante le formule:

$$
C_{T_{\mathrm{h}}}^{\mathrm{o}}=\frac{3,38}{! \mathrm{l}} 10^{-2} \quad C_{\mathrm{U}}^{\mathrm{o}}=\frac{1,02}{\mu} \mathrm{J} 0^{--2}
$$

Le corrispondenti concentrazioni $C_{10}^{\prime \prime}, C^{\prime \prime}{ }_{\text {Ra }}$ ehe darebhero luogo alle distribuzioni $\mathrm{II}^{\prime}$ e $\mathrm{II}^{\prime \prime}$ sono quelle stesse che competerebhero all'Ionio e al Ra rispettivamente se essi fossero in equilibrio con $U$ di concentrazione $C_{\mathrm{U}}^{0}$.

Nel seguito assumeremo queste concentrazioni come unità convenzionali per il Th, l'U, Io, Ra.

Indicando con $A$ la superficie dell'emulsione esplorata in $\mathrm{cm}^{2}$, con $t$ il tempo di esposizione in sec, $d$ la densità del materiale in $\mathrm{gr} / \mathrm{cm}^{3}$, la concentrazione assoluta di Th in gr/gr di materia, è data da:

$$
r_{\mathrm{Th}}=\frac{\bar{a} C_{\mathrm{Th}}^{\mathrm{o}}}{\bar{A} t d}
$$

Formule analoghe si hanno per le concentrazioni degli altri elementi.

Evidentemente per applicare la formula [5] è necessario poter determinare il valore di $\mu$, rapporto tra percorso delle $\alpha$ nella materia e percorso in aria. Nell'ipotesi di Bragg e Kleeman ( $\left.{ }^{+}\right)$si ha:

dove

$$
\mu=\frac{d_{\text {aria }}}{d_{\text {mat }}} \frac{\psi_{\text {mat }}}{\psi_{\text {'rria }}}=3 ; 19 \times 10^{-4} \frac{\psi_{\text {mat }}}{\varrho_{\text {mat }}}
$$

$$
\eta_{\text {mat }}^{\prime}=\sum_{1}^{\mathrm{n}} a_{\mathrm{i}} \sqrt{W_{\mathrm{i}}}
$$

con $a_{i}$ percentuale dell'elemento considerato costituente il materiale e $W_{1}$ corrispondente peso atomico. Per la nostra lava è stata calco-

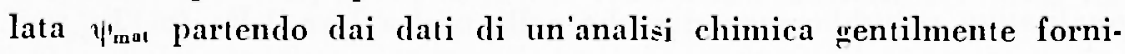
taci dal prof. Ponte unitamente al campione di lava; per essa si ha:

$$
\psi_{\text {'mat }}=4,5
$$

Nel caso presente data la natura del campione (polvere) si è preferito applicare la formula [5] ai dati riguardanti la stessa lava esposta in sezione levigata, per la ragione che per quest'ultima ha 
senso parlare di una densità e di una superficie esposta. Dai dati del lavoro già citato $\left({ }^{1}\right)$ e prendendo per $a$ e $b$ i valori già determinati, si ottiene:

$$
C_{\mathrm{Th}}=1,58 \cdot 10^{\cdot \bar{\top}} \text { grammi di Th per grammo di lava, }
$$

e nell'ipotesi che l'Uranio fosse in equilibrio:

$$
C_{\mathrm{u}}=0,7 \cdot 10^{-6} \stackrel{\mathrm{gr} / \mathrm{gr}}{\mathrm{r}}
$$

In quest'ultima ipotesi dell'equilibrio della famiglia dell' $U$, si ha:

$$
C_{\mathrm{v}} / C_{\mathrm{Tu}}=0,04
$$

Partendo dalle formule date da I. Curie ( $\left.{ }^{9}\right)$, che del resto riguardano un caso particolare rispetto a quelle applicate sopra e valide sempre solo per la statistica delle lunghezze totali, si ottiene un valore lecrgermente diverso:

$$
C_{\mathrm{U}} / C_{\mathrm{Tu}}=0,05(\mathrm{~m})
$$

Per esaminare ora il problema dell'equilibrio si dovrebbe fare il confronto della parte di spettro sperimentale di ascisse minori di 3,21 con le corrispondenti parti degli spettri teorici, cioè analiticanente si dovreblue cercare di determinare i coefficienti $b, b^{\prime}, b^{\prime \prime}$ dell'equazione:

$$
f(r)-a \varphi(r)=b \%(r)+b^{\prime} \chi^{\prime}(r)+b^{\prime \prime} \%^{\prime \prime}(r) \quad(r<3.21)
$$

Poiché nel caso della nostra lava la concentrazione degli elementi della famiglia dell'U rispetto a quelli del Th è risultata troppo bassa, abljiamo avuto troppo poche tracce corte utilizzabili per poter tentare una determinazione dei coefficienti, perché le tracce corte sono in massima parte dovute ad elementi della famiglia dell'U. L'andamento qualitativo potrebloe suggerire che nella nostra lava il Ra (o l'Io) non sia in equilibrio con l'U ma che questo ultimo abbjia una concentrazione minore di quella che avreblye se fosse in equilibrio; però a causa del piccolo numero di tracce côrte imputabili a questi elementi, non

(**) Nel caso presente è difficile calcolare l'errore sul rapporto Uranio-Torio, si può solo dire che l'accordo tra i 2 valori ricavati con netodi diversi è buono. 
crediamo di avere dati sufficienti per potere trattare quantitativamente il problema.

Notiamo a questo proposito clie la valutazione dell'esistenza o meno dell'equilibrio della famiglia dell'Uranio esige una conoscenza molto esatta del rapporto teorico tra il numero di tracce lunghe ed il numero di tracce corte; d'altra parte, questo rapporto è certamente influenzato, ed in misura notevole, dalla non proporzionalità tra i percorsi nel materiale e nella gelatina di particelle della stessa energia. Agriungiamo che tale correzione può mascherare completamente l'andamento effetlivo di quella parte dello spettro che corrisponde ai valori più bassi del percorso, e quindi dare risultati errati per quanto riquarda l'equilibrio.

Ringraziamo il prof. G. Ponte per averci forniti i campioni da noi studiati e per averci comunicati i risultati dell'analisi chimica che ci ha permesso il calcolo di l'mat.

I nostri ringraziamenti vanno purc rivolti alla Direzione Generale del Banco di Sicilia che con una sua elargizione ha contribuito in parte alla spesa per l'acquisto di un microscopio.

Palermo - Istituto di Fisica dell'Università - Maggio 1953.

\section{RIASSUNTO}

Nel presente lavoro vengono esposti i risultati di una ricerca sul contenuto di elementi delle due famiglie naturali dell'Uranio e del Torio in un campione di lava dell'Etna.

La tecnica impiegata è quella delle lastre sensibili per fisica nucleare.

Si espongono $i$ criteri usati per la determinazione del rapporto tra il contenuto di Uranio e quello di Torio nel caso dell'equilibrio della famiglia dell'Vranio. Quest'ultima questione viene trattata e discussa dal punto di vista generale e analitico: si traccia un metodo per risalire dal confronto tra lo spettro sperimentale dai ranges delle tracce a in emulsione con quello teorico, alla esistenza o meno dell'equilibrio radioattivo. 


\section{$S U M M A R Y$}

The results of a research on the content of elements of Uranium and Thorium family in a lava sample from Mt. Etna are presented.

The nuclear emulsion tecnique has been used.

The principles adopted for the determination of ratio of Uranium to Thorium content in the hypothesis of equilibrium are exposed. The last question is discussed from a general and analytical view, a procedure is shown in order to obtain, from a comparison of experimental spectrum of the alpha tracks in emulsion with theoretical spectrum, the eventual presence of radioactive equilibrium.

\section{BIBLIOGRAFIA}

(1) L. Barbera, M. Curatoln, M. Indovive Adomio, M. Saxtangelo: Ann. Geof., 5, 603 (1952).

(2) Van Stijvesdael: Bull. Gen. Phỵs. Nucl., n. 28, 1951; G. Imbò-L. Cisertano: Ann. Geof., V, 1952; D. PaLuvio: Ann. Geof., in corso di stampa.

(3) R. Coppens: Bull. Soc. Franc. Min. Crist., 73, 217 (1950).

(4) H. Yagoda: Radionchive Measurements with Nuclear Emulsion (N. Y. 1949).

(5) E. Picciotro: Bull. Centre Plys. Nucléaire Uin. Libre Bruxelles, n. 33 (1952).

S. Milone Tamberivo, A. Stella: N. Cim, IX, 253 (1952).

(6) M. Bocandot e B. KocdiJs: Phys. Rev., 87, 1129 (1952).

(7) H. V. Buttlan e F. G. Holtemmas: Geoc. et Cosmoc. Actia, 2, 43 (1951).

(8) Landolt-Bonnsteiv: Zahlenuerte und Funhtionen ans Phvsik 6 Auflage.

(9) I. Curie: Journ. de Phys. et Rad., 11, 633 (1946). 